\title{
The lag phase rather than the exponential- growth phase on glucose is associated with a higher CAMP level in wild-type and CAPK- attenuated strains of the yeast Saccharomyces cerevisiae
}

\author{
Pingsheng Ma, ${ }^{1}$ Teresa Gonçalves, ${ }^{2}$ António Maretzek, ${ }^{3}$ \\ Maria C. Loureiro Dias ${ }^{2}$ and Johan M. Thevelein ${ }^{1}$
}

Author for correspondence: Johan M. Thevelein. Tel: +32 16321507 or 321500 . Fax : +32 16321979 .
e-mail : johan.thevelein@ @io.kuleuven.ac.be

1 Laboratorium voor Moleculaire Celbiologie, Katholieke Universiteit Leuven, Kardinaal Mercierlaan 92, B-3001 Leuven-Heverlee, Flanders, Belgium

2 Laboratorio de Microbiologia, Instituto Gulbenkian de Ciência, Ap. 14, 2781 Oeiras Codex, Portugal

3 Instituto de Tecnologia Quimica e Biologica/ Instituto de Biologia Experimental e Tecnologica, Universidade Nova de Lisboa, Apartado 127, 2780 Oeiras, Portugal

In the yeast Saccharomyces cerevisiae several phenotypic properties controlled by CAMP-dependent protein kinase (CAPK) are indicative of high CAPK activity during growth on glucose and low activity during growth on non-fermentable carbon sources and in stationary phase. It has been a matter of debate whether the apparently higher activity of CAPK in cells growing on glucose is due to a higher CAMP level or to an alternative mechanism activating CAPK. The CAMP level during diauxic growth of yeast cells in cultures with different initial glucose levels and different initial cell densities has been reinvestigated and the previously reported twofold increase in CAMP during growth initiation has been confirmed. However, this increase was transient and entirely associated with the lag phase of growth. The initiation of exponential growth on glucose was associated with a decrease in the CAMP level and there was no correlation between this decrease in CAMP and the depletion of glucose in the medium. In mutants defective in feedback inhibition of CAMP synthesis, resuspension of exponential-phase glucosegrown cells in glucose medium caused an extended lag phase during which a huge, transient accumulation of CAMP occurred. The latter required the presence of glucose and nitrogen, but not phosphate or sulfate, and was not due to intracellular acidification, as shown by in vivo "11P-NMR spectroscopy. The initiation of exponential growth on glucose was also associated in this case with a decrease in CAMP rather than an increase. This behaviour was also observed in strains with attenuated catalytic subunit activity and lacking the regulatory subunit and even in strains without catalytic subunits of cAPK. This might indicate that other mechanisms are able to cause down-regulation of CAMP synthesis in a way mimicking feedback inhibition. Transfer of glucosegrowing cells of wild-type or CAPK-attenuated strains to a nitrogen starvation medium resulted in an increase in the CAMP level rather than a decrease. The results indicate that the apparent changes in CAPK activity in vivo during diauxic growth on glucose and during nitrogen starvation cannot be explained on the basis of changes in the CAMP level.

Keywords: cAMP-dependent protein kinase, signal transduction, trehalose, glycogen, stress resistance

Abbreviation: CAPK, CAMP-dependent protein kinase. 


\section{INTRODUCTION}

In the yeast Saccharomyces cerevisiae several phenotypic characteristics are known to be affected drastically by the activity of cAMP-dependent protein kinase (cAPK). Typical examples include the level of trehalose and glycogen, inherent and acquired stress resistance and the expression of 'STRE'-controlled genes like CTT1 (catalase) and $S S A 3$ (Hsp70). In yeast mutants with a reduced cAMP level or with reduced activity of cAPK all these parameters are up-regulated compared to wild-type cells, whereas in yeast mutants with an elevated cAMP level or with enhanced activity of cAPK these parameters are down-regulated. A very similar difference in the same phenotypic properties is observed between cells growing fermentatively on glucose or related rapidly fermented sugars and cells growing respiratively on nonfermentable carbon sources like glycerol or ethanol. The former display a 'high-cAMP phenotype' while the latter display a 'low-cAMP phenotype' (Tatchell, 1993; Thevelein, 1994).

Two different views have been proposed to explain the striking difference in cAPK-controlled properties between cells growing on glucose and those growing on non-fermentable carbon sources. The most straightforward view proposes that the cAMP level is higher in cells growing on glucose and that the resulting higher activity of cAPK is responsible for the observed phenotypic differences (Boy-Marcotte et al., 1987; Dumont et al., 1989; Engelberg et al., 1989; François et al., 1987; Gibbs \& Marshall, 1989). This view is supported by the well-known stimulating effect of glucose on cAMP synthesis observed immediately after addition of glucose to cells growing on a non-fermentable carbon source (Beullens et al., 1988; Eraso \& Gancedo, 1985; Thevelein \& Beullens, 1985; van der Plaat, 1974). cAMP levels during diauxic growth were measured by François $e t$ al. (1987) and Russell et al. (1993). François et al. (1987) reported that cAMP decreased parallel to the disappearance of glucose in the medium and stayed constant after glucose depletion. Russell et al. (1993) reported that cAMP dropped during the early fermentative phase of growth to reach a minimum at the diauxic shift. Afterwards the cAMP level rose somewhat but never regained the level present during the fermentative phase. In both cases the largest difference in the cAMP level was slightly more than a factor of two. Closer inspection of the data reported in both papers, however, reveals that the highest cAMP level was present during the initial lag phase of the culture. During subsequent initiation of exponential growth on glucose the cAMP level dropped and the further decrease associated with the diauxic transition was very small and does not appear to be significant. This raises the question whether the drop in cAMP is really related to the disappearance of glucose in the medium or whether it is associated, for instance, with exit from lag phase.

We have proposed that the cAMP level in cells growing on glucose is essentially the same as in cells growing on non-fermentable carbon sources and that the difference in the apparent level of cAPK activity is caused by another pathway which triggers activation of the free catalytic subunits of cAPK in a novel cAMP-independent way. We have called this pathway the 'Fermentable-growth-medium-induced pathway' (Thevelein, 1991, 1994). This suggestion was based on the glucose-repressible character of glucose-induced activation of cAMP synthesis (Argüelles et al., 1990; Beullens et al., 1988; Dumortier et al., 1995), the observation that other nutrients in the presence of glucose can trigger activation of trehalase without effect on cAMP (Hirimburegama et al., 1992) and by the demonstration that several targets of cAPK are still regulated during diauxic growth in strains lacking the regulatory subunit of cAPK (Belazzi et al., 1991; Cameron et al., 1988; Durnez et al., 1994).

In an attempt to resolve these opposing views we have re-investigated the cAMP level in a wild-type strain during growth on glucose, using cultures with different initial glucose levels and cell densities. In addition, we have made use of strains with attenuated activity of cAPK. In S. cerevisiae the genes TPK1, TPK2 and TPK3 encode the catalytic subunits of CAPK, whereas the $B C Y 1$ gene encodes the regulatory subunit (Toda et al., $1987 a, b)$. Yeast strains with deletions of two of the TPK genes and a partially inactivating mutation in the third TPK gene display strongly reduced activity of cAPK. Such strains were found to contain highly elevated levels of cAMP, indicating the existence of strong feedback inhibition of cAPK on cAMP synthesis in wild-type strains (Nikawa et al., 1987). Glucose and other fermentable sugars cause rapid and huge increases in the cAMP level in cells of such strains (Mbonyi et al., 1990). Deletion of the three TPK genes is lethal in a wild-type strain, but this lethality can be rescued by additional deletion of the YAK1 gene which encodes a protein kinase with unknown function (Garret \& Broach, 1989). We now show that such quadruple deletion mutants also display highly elevated cAMP levels. Using this set of strains we show that the lag phase of growth is associated with a transient increase in cAMP, the initiation of exponential growth on glucose is associated with a decrease in CAMP and that there is no correlation between the decrease in the cAMP level and the depletion of glucose in the medium.

\section{METHODS}

Yeast strains and growth conditions. The following isogenic Saccharomyces cerevisiae strains were used in this study: DC124 (Mata his4 leu 2 ura3 trp1 ade8 can1) (isogenic to SP1), RS13-58A-1 (MATa his3 leu2 ura3 trp1 ade8 tpk $1^{\text {w1 }}$ tpk2::HIS3 tpk3::TRP1 bcy1::LEU2), RS13-7C-1 (MATa his3 leu2 ura3 trp1 ade8 tpk1::URA3 tpk2 ${ }^{\mathrm{w} 1}$ tpk3::TRP1 bcy1::LEU2), RTF3.1-1 (MATa bis3 leu2 ura3 trp1 ade8 tpk1::URA3 tpk2::HIS3 tpk3 ${ }^{\text {w1 }}$ bcy1::LEU2) (Nikawa et al., 1987), SGP406 (MATa leu2-3,112 trp1 ura3-52 his3 tpk1::URA3 tpk2::HIS3 tpk3:: TRP1 yak1::LEU2), SGP400 (MATa leu2-3,112 trp1 ura3-52 his3 yak1::LEU2) (Garret \& Broach, 1989). The wild-type strain was grown on SD glucose medium (Sherman et al., 1986) with an initial glucose level of 
1,2 and $4 \%(\mathrm{w} / \mathrm{v})$ and an initial cell density of 0.5 (measured as $\left.\mathrm{OD}_{600}\right)$. In addition the wild-type strain was also grown with an initial glucose level of $2 \%$ in the medium and an initial cell density of $0 \cdot 1\left(\mathrm{OD}_{600}\right)$. The cells of the strains with attenuated cAPK activity (and the wild-type strain as control) were grown on SD glucose medium ( $2 \%$ glucose) to exponential phase, harvested by centrifugation, washed with cold water and resuspended in fresh SD glucose medium at an $\mathrm{OD}_{600}$ of $1 \cdot 0$. All cultures were grown in shake flasks in a gyratory incubator at 200 r.p.m. from starter cultures grown on SD glucose medium to exponential phase. Glucose was added 10 min after inoculation of the cells in SD medium. The first sample (time 0 ) was taken $10 \mathrm{~min}$ after inoculation, just before the addition of glucose.

Determination of CAMP content. Samples for cAMP determination were taken as indicated. Extraction of the cells and determination of the cAMP level were carried out as described previously (Thevelein et al., 1987a).

In vivo ${ }^{3}$ P-NMR spectroscopy. The NMR experiments could not be performed under exactly the same conditions as the cAMP determinations due to technical reasons. However, the changes observed in the glycolytic intermediates and the intracellular $\mathrm{pH}$ by ${ }^{31} \mathrm{P}-\mathrm{NMR}$ spectroscopy are indicative of normal initiation of glucose metabolism in all the strains used. The cells were grown under the same conditions as for the cAMP experiments, harvested and resuspended in $25 \mathrm{mM}$ Tris/citrate buffer, $\mathrm{pH} 5.5$. For each in vivo ${ }^{31} \mathrm{P}-\mathrm{NMR}$ experiment about $200 \mathrm{mg}$ dry wt of cells was resuspended in $4 \mathrm{ml}$ of the same buffer. NMR spectra were recorded at $25^{\circ} \mathrm{C}$ using a Bruker AMX-300 or an AMX-500 spectrometer, operating at 121.5 or $202.46 \mathrm{Mhz}$, respectively. NMR tubes $(10 \mathrm{~mm})$ were used in a broadband probe. An efficient mixing of the cell suspension was achieved by using an air-lift system (Santos \& Turner, 1986). Spectra were acquired with pulses of about $45^{\circ}$, a repetition time of $0.5 \mathrm{~s}$ and 360 scans per spectrum. Chemical shifts were referenced to $85 \% \mathrm{H}_{3} \mathrm{PO}_{4}$ at 0 p.p.m. The resonance of MDP (methylenediphosphonate) contained in a capillary tube, calibrated against $85 \% \mathrm{H}_{3} \mathrm{PO}_{4}$, was used as the chemical shift standard. To calculate $\mathrm{pH}$ values from the chemical shift of the inorganic phosphate, a calibration curve was constructed using a cell-free extract. Peaks were assigned according to the literature (Gage $e t$ al., 1984; Loureiro-Dias \& Santos, 1990).

Reproducibility of results. All experiments were repeated at least twice with consistent results. Representative results are shown.

\section{RESULTS}

The cAMP level in the wild-type strain DC124 (isogenic to SP1) was measured during growth on SD glucose medium. Three different initial glucose levels were used: 1,2 and $4 \%$. In addition, a fivefold-lower initial cell density was also used for a culture with $2 \%$ glucose. Fig. 1 shows the $\mathrm{OD}_{600}$ of the cultures, the glucose level in the medium and the cAMP level in the cells. The results show that growth of the cultures is initially associated with a higher cAMP level. The difference is about twofold, in agreement with earlier reports (François et al., 1987; Russell et al., 1993). However, the cAMP increase is clearly transient and it is entirely associated with the lag phase of the cultures (from 0 to about $2-3 \mathrm{~h}$ ). The initiation of exponential growth on glucose

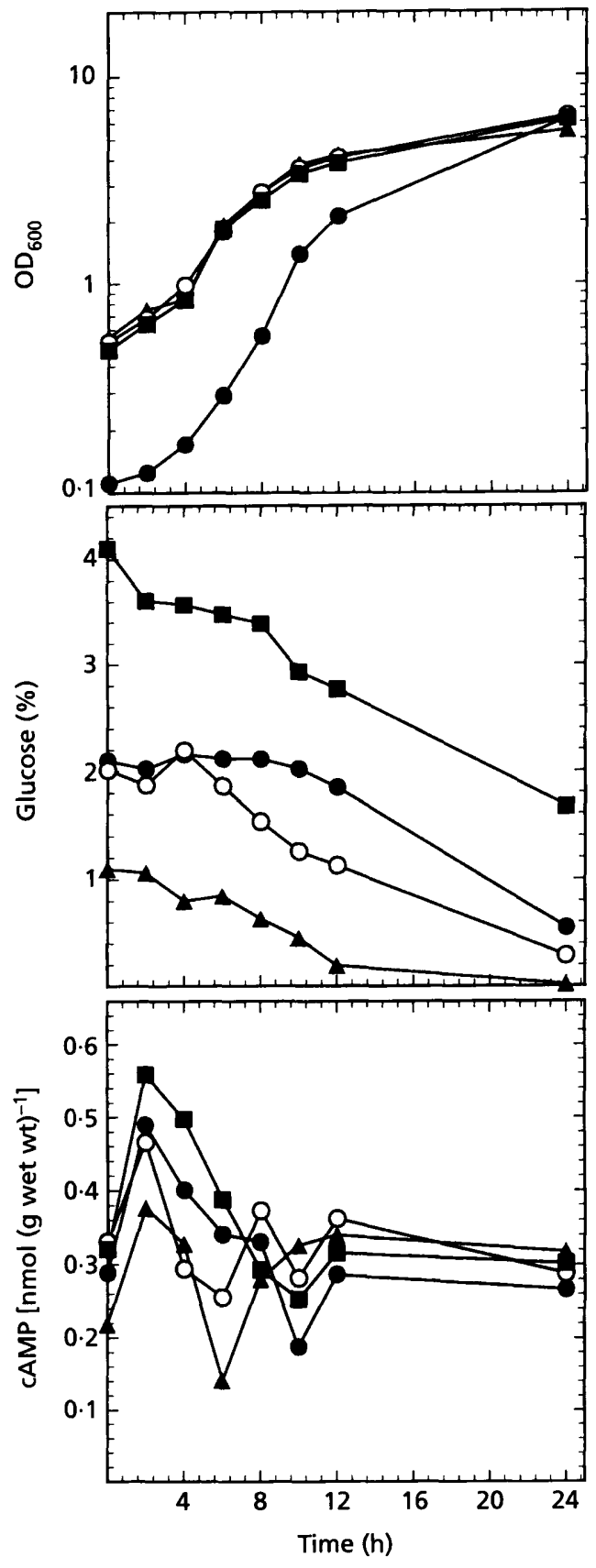

Fig. 1. Cell density, glucose level in the medium and intracellular CAMP content during diauxic growth of cultures of a wild-type strain. Initial cell density $0.5\left(O_{600}\right)$ and initial glucose level $1 \%(\Delta), 2 \%(O)$ and $4 \%(\mathbb{D})$; initial cell density $0.1\left(O D_{600}\right)$ and initial glucose level $2 \%(0)$.

(at about 3-5 h) is associated with a decrease in the cAMP level, which does not coincide precisely, however, with the increase in $\mathrm{OD}_{600}$ in the different cultures. During subsequent growth the cAMP level remains approximately constant. When the initial glucose level was reduced to $1 \%$ or doubled to $4 \%$, a very large difference in the glucose levels remaining in the cultures 


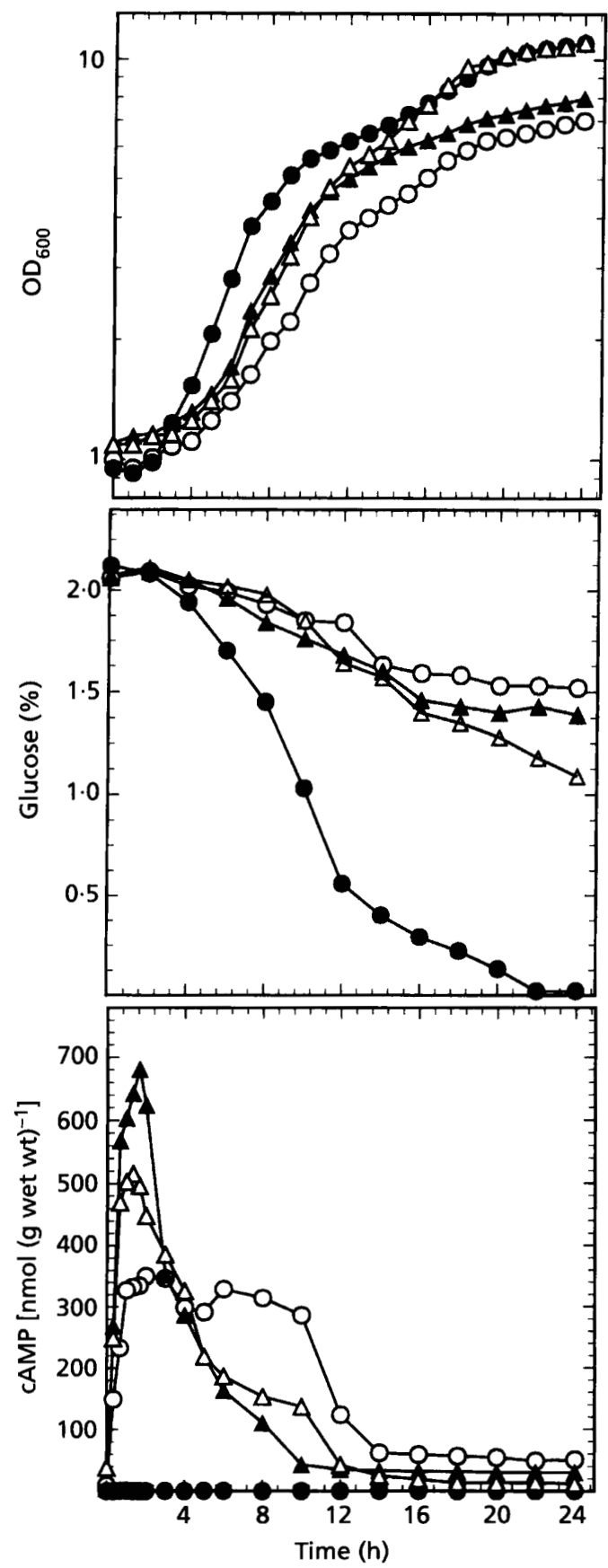

Fig. 2. Exponential-phase SD-glucose-grown cells were harvested and resuspended in fresh SD glucose medium after which cell density, glucose level in the medium and intracellular CAMP content of the cells were monitored. Strains: wild-type (O), a strain with reduced CAPK activity (S18-1D, tpk $1^{\mathrm{w} 1}$ tpk2 tpk $3 \Delta)(O)$, a strain with reduced and constitutive CAPK activity (RS13-58A-1, tpk $1^{\mathrm{w} 1}$ tpk2 $2 \Delta$ tpk3 $\Delta$ bcy1 $\Delta$ ) ( $\Delta$ ) and a strain which entirely lacks the genes encoding the catalytic subunits

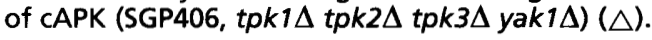

at the different time points was observed. However, in all cases the transient increase in the cAMP level was very similar and entirely associated with the lag phase of the cultures. There was a tendency for a somewhat higher transient cAMP increase during the lag phase with increasing glucose levels in the medium (Fig. 1). When the initial cell density was reduced fivefold in a culture with an initial glucose level of $2 \%$, the increase in $\mathrm{OD}_{600}$ and the usage of the glucose in the medium was clearly retarded compared to the control culture. During the first $8 \mathrm{~h}$ the glucose level in the medium did not decrease noticeably. However, the course of the cAMP level again was very similar to that of the other three cultures (Fig. 1).

In this study we also made use of yeast strains with partially or completely defective cAPK activity. Such strains display reduced feedback inhibition by cAPK of cAMP synthesis and as a result they have much higher and also much more variable cAMP levels than the wildtype strains (Nikawa et al., 1987; Mbonyi et al., 1990). To avoid as much as possible that any increase in the cAMP level could be attributed to adaptation of such strains to the glucose-containing medium, as previously observed (Mbonyi et al., 1990), we grew the cells first on SD glucose medium to exponential phase, after which they were harvested, washed with cold water and resuspended in fresh SD glucose medium. The following strains were used: a strain with a partially inactivating point mutation in TPK1 and a deletion of TPK2 and $T P K 3$, which displays reduced activity of cAPK $\left(t p k 1^{\mathrm{w} 1}\right.$ $t p k 2 \Delta t p k 3 \Delta)$, the same strain but with an additional deletion of $B C Y 1$, which displays reduced and constitutive activity of cAPK $\left(t p k 1^{\mathrm{w1}} t p k 2 \Delta t p k 3 \Delta b c y 1 \Delta\right)$, and a strain with a deletion of the three TPK genes and an additional deletion of YAK1 to overcome the lethality caused by the triple deletion ( $t p k 1 \Delta t p k 2 \Delta t p k 3 \Delta$ yak1 $\Delta$ ). In addition to the wild-type strain, the yak1 $1 \Delta$ strain was also used as a control.

The three strains with defective cAPK activity displayed a much longer lag phase than the wild-type strain upon resumption of growth (Fig. 2). They also consumed the glucose in the medium much more slowly than the wildtype strain (Fig. 2). In the three strains glucose consumption paralleled the increase in $\mathrm{OD}_{600}$ with the $t p k 1^{\mathrm{w} 1} B C Y 1$ strain being the slowest and the $t p k 1-3 \Delta$

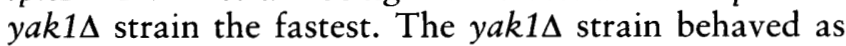
the wild-type strain (results not shown). Two other strains with reduced, constitutive activity of cAPK,

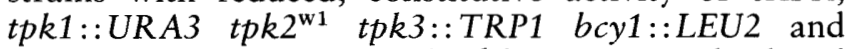
tpk1::URA3 tpk2::HIS3 tpk3 $3^{\mathrm{w} 1}$ bcy1 ::LEU2, displayed a similar lag phase before growth resumed (results not shown). In the $t p k 2^{\mathrm{w} 1}$ strain the lag phase was somewhat shorter which fits with the previous observation that this strain appears to have the highest remaining protein kinase activity (Durnez et al., 1994). During the extended lag phase preceding exponential growth the three strains with defective CAPK activity showed a huge transient increase in the cAMP level (Fig. 2). The maximum cAMP level attained was about 500- to 1500 -fold higher than the maximum level observed during the lag phase in wild-type cells. This maximum level was rather variable between different experiments but the time dependence of the changes was more constant. The decrease in the cAMP level always coincided approxi- 


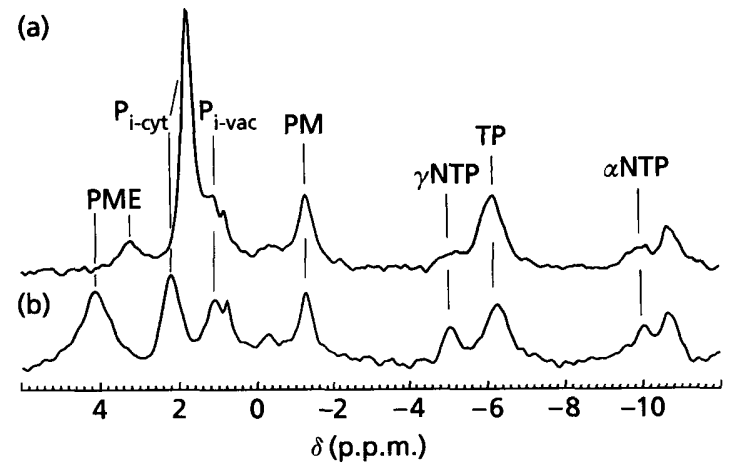

Fig. 3. In vivo ${ }^{31}$ P-NMR spectra of cells of the tpk1 tpk2 $\Delta$ tp $k 3 \Delta$ yak $1 \Delta$ strain (a) before and (b) 3 min after the addition of $50 \mathrm{mM}$ glucose (100 $\mu \mathrm{l}$ of a $2 \mathrm{M}$ glucose solution). PME, phosphomonoesters; $P_{i-c y t}$ cytoplasmic phosphate; $P_{i-v a c}$ vacuolar phosphate; PM, phosphomannan; NTP, nucleotide triphosphate; TP, terminal phosphate of polyphosphate.

mately with the end of the lag phase. In the $t p k 1^{\mathrm{w} 1} B C Y 1$ strain, which displayed the longest lag phase, this transient hyperaccumulation of cAMP also lasted longest (Fig. 2). (The drop at $4 \mathrm{~h}$ in Fig. 2 was not reproducible.) The drop in the cAMP content following the initial increase was very clearly not related to the depletion of glucose in the medium (Fig. 2). The two other strains with reduced constitutive activity of cAPK, tpk1::URA3 tpk2 $2^{\mathrm{w} 1}$ tpk3::TRP1 bcy1::LEU2 and tpk1::URA3 tpk2::HIS3 tpk3 ${ }^{\mathrm{w} 1}$ bcy1 ::LEU2, displayed the same transient increase except that in the former strain the increase in the cAMP level was smaller, consistent with its higher protein kinase activity (results not shown). The transient increase in the cAMP level was not affected by addition of the respiration inhibitor Antimycin A (results not shown).

The extended lag phase of the cAPK-defective mutants indicates that they have a problem in continuing growth even after a relatively slight disturbance in the growth conditions. This problem might be situated, for example, in metabolism or in cell cycle progression. If the problem was situated in glucose metabolism, the transient increase in the cAMP level could be due to a transient intracellular acidification occurring during this extended lag phase. Wild-type cells are known to display a very short-lived intracellular acidification upon addition of glucose (den Hollander et al., 1981; Thevelein et al., 1987b). This transient acidification is due to the generation of protons in glucose catabolism and is counteracted by the plasma membrane $\mathrm{H}^{+}$-ATPase, of which the activity depends on efficient generation of ATP. Glucose addition to wild-type cells causes higher activity of the plasma membrane $\mathrm{H}^{+}$-ATPase because of the increase in the ATP level and because of posttranslational activation of the enzyme (Serrano, 1983). Therefore glucose addition to wild-type cells is invariably associated with a clear increase in the intracellular $\mathrm{pH}$ after the very short-lived initial decrease.

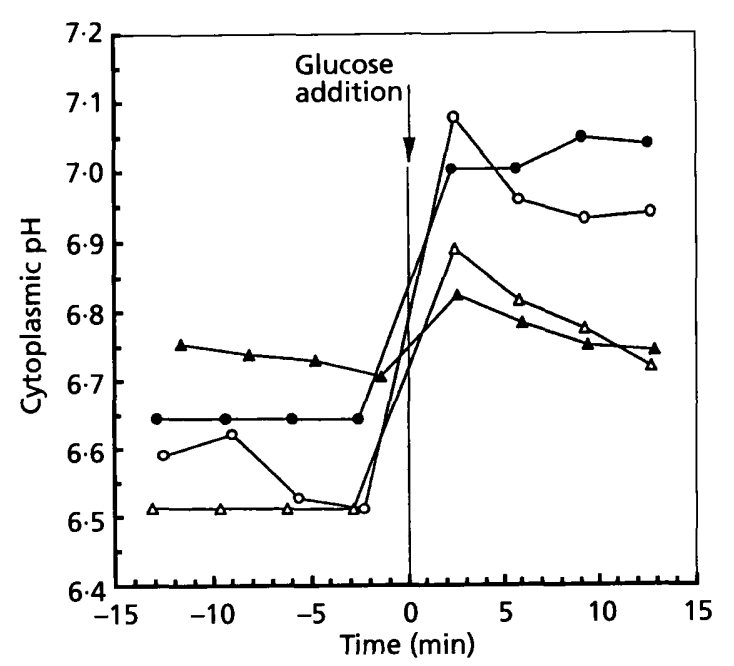

Fig. 4. Cytoplasmic $\mathrm{pH}$, as measured by in vivo ${ }^{31} \mathrm{P}-\mathrm{NMR}$ spectroscopy, as a function of time after glucose addition. The $\mathrm{pH}$ values were determined from the chemical shifts of the resonances of $P_{i-c y t}$ using a calibration curve. Glucose $(50 \mathrm{mM})$ was added at time 0 . Spectra were accumulated before and after glucose addition. Strains: wild-type (O), a strain with

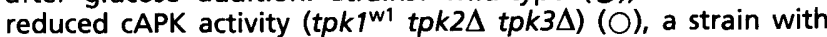

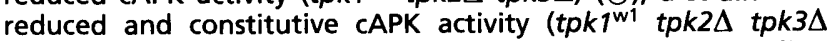
bcy $1 \Delta)(\triangle)$ and a strain which entirely lacks the genes encoding the catalytic subunits of CAPK (tpk $1 \Delta$ tpk $2 \Delta$ tpk3 $\Delta$ yak $1 \Delta)(\Delta)$.

To investigate whether the large transient increase in the cAMP level in the yeast strains defective in cAPK could be due to an extended intracellular acidification of the cells, we have measured, by means of in vivo ${ }^{31} \mathrm{P}-\mathrm{NMR}$ spectroscopy, the intracellular $\mathrm{pH}$ in such strains as a function of time after addition of glucose. Representative ${ }^{31}$ P-NMR spectra are shown in Fig. 3 for cells of the $t p k 1 \Delta t p k 2 \Delta t p k 3 \Delta$ yak1 1 strain before and $3 \mathrm{~min}$ after glucose addition. From the position of the phosphate peak in the ${ }^{31} \mathrm{P}-\mathrm{NMR}$ spectrum the cytoplasmic $\mathrm{pH}$ was determined as a function of time after glucose addition (den Hollander et al., 1981). The results are shown in Fig. 4 for the wild-type, $t p k 1^{\mathrm{w} 1} t p k 2 \Delta t p k 3 \Delta$,

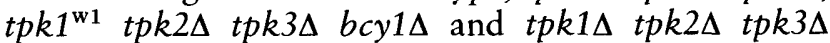
yak1 $1 \Delta$ strains. They clearly show that the strains with partially or completely defective cAPK activity raise their intracellular $\mathrm{pH}$ within a few minutes after glucose addition in a very similar way as observed for the wildtype strain. Both the rate and the extent of the increase did not seem to be affected strongly by the defects in cAPK activity. Similarly, the increase in the ATP and sugar phosphate levels was not significantly different between the wild-type strain and the other strains (results not shown). Hence, this large, transient hyperaccumulation of cAMP cannot be ascribed to a general metabolic weakness due to the defective cAPK activity.

Because of technical reasons the in vivo ${ }^{31} \mathrm{P}-\mathrm{NMR}$ experiments could not be performed under exactly the same conditions as the cAMP measurements. In spite of this, we feel that the results indicate that the cAPK- 


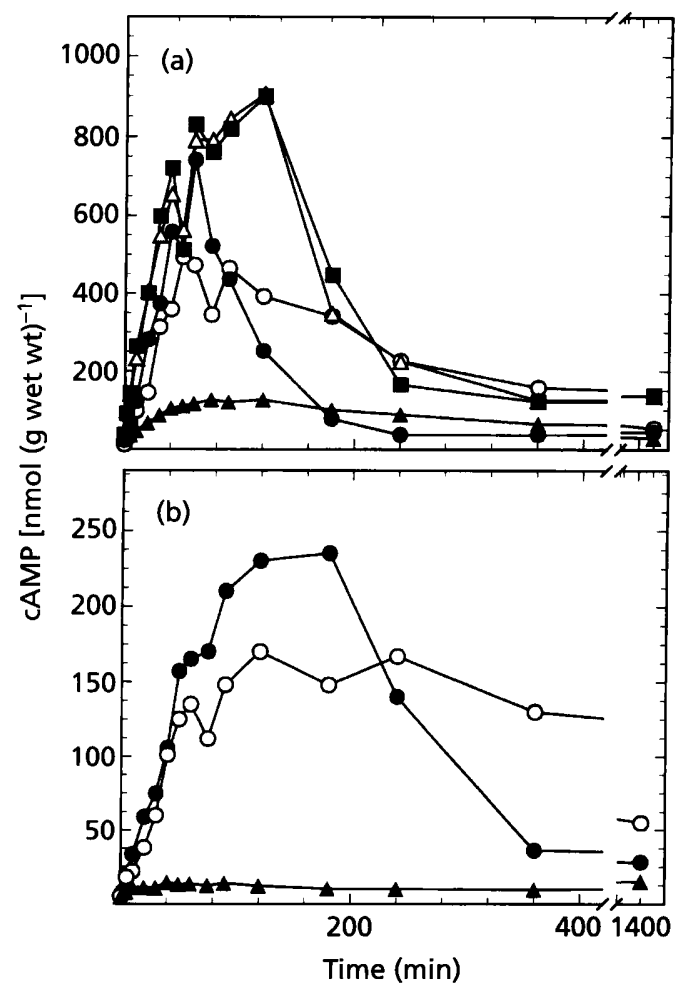

Fig. 5. Transient hyperaccumulation of CAMP during the lag phase. (a) Strain with reduced and constitutive CAPK activity

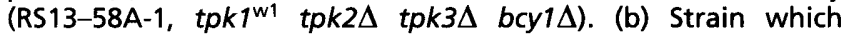
entirely lacks the genes encoding the catalytic subunits of CAPK

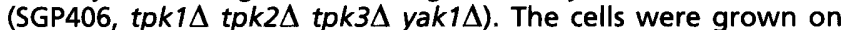
SD glucose to exponential phase, harvested and resuspended in fresh SD glucose medium in the absence $(O)$ or presence $(O)$ of cycloheximide or transferred to SD glucose medium lacking either nitrogen $(\Delta)$, phosphate $(\triangle)$ or sulfate $(\boldsymbol{D})$.

defective strains show normal initiation of glucose catabolism and enhancement of intracellular $\mathrm{pH}$ and that in all likelihood they also display such behaviour under standard growth conditions.

When cells of the mutants with reduced feedback inhibition of cAMP synthesis were transferred to fresh SD glucose medium lacking nitrogen the hyperaccumulation of cAMP was strongly reduced. This is shown in Fig. 5(a) for the strain displaying reduced, constitutive activity of cAPK, $t p k 1^{\mathrm{w1}} t p k 2 \Delta t p k 3 \Delta b c y 1 \Delta$, and in Fig. 5 (b) for the $t p k 1-3 \Delta$ yak $1 \Delta$ strain. The transfer to nitrogen starvation medium, however, still caused a pronounced increase in the cAMP level rather than the decrease which would be expected on the basis of the change in CAPK-controlled characteristics of wild-type cells when transferred to the same nitrogen starvation condition. In the strain displaying reduced, constitutive

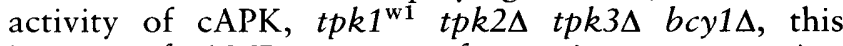
increase of cAMP upon transfer to nitrogen starvation medium was also transient but the cAMP level started to drop only about $1 \mathrm{~h}$ later than when the cells were transferred to full SD glucose medium (Fig. 5). Transfer of the cells of this strain to SD glucose medium lacking either phosphate or sulfate resulted in the same transient hyperaccumulation as during transfer to complete $\mathrm{SD}$ glucose medium except that the cAMP level also started to drop about $1 \mathrm{~h}$ later than in the full medium (Fig. 5). Addition of cycloheximide did not affect the rate of the initial increase in cAMP for both strains but reduced the maximum extent of the increase and slowed down the decrease to a variable extent in different experiments (Fig. 5 and results not shown).

When the cells were transferred to SD medium without glucose, the cAMP increase was very small. The presence of $5 \mathrm{mM}$ glucose was enough to attain $50 \%$ of the increase with $100 \mathrm{mM}$ (results not shown). Together with the strong requirement for nitrogen this seems to support that only a lag phase preceding growth is associated with the huge transient cAMP increase. With cells grown and resuspended in SD glycerol medium with or without nitrogen source no significant cAMP increase was observed, further supporting the importance of the presence of a fermentable sugar in the medium (results not shown).

We have also performed a similar experiment with a wild-type strain. Although the variation in the cAMP level was very small compared to the feedback-inhibition-defective strains, an initial very short-lived increase could also be detected upon transfer of the cells to complete SD glucose medium (results not shown). Transfer to SD glucose medium lacking nitrogen also resulted in a transient increase in the cAMP level in the wild-type strain rather than an immediate decrease. On the other hand, during prolonged nitrogen starvation the cAMP level dropped to a somewhat lower level than in the cells on complete SD glucose medium. The difference, however, was at most a factor two (results not shown).

\section{DISCUSSION}

Our results on the cAMP level during diauxic growth confirm earlier results (François et al., 1987; Russell et al., 1993) that the cAMP level is highest at the initiation of the growth curve. However, they clearly contradict the conclusion that the decrease in the cAMP level correlates with the depletion of glucose in the medium and that therefore growth on glucose is associated with a higher cAMP level. Our results show that the lag phase of growth is specifically associated with an increase in the cAMP level and that this level decreases again at or before the actual initiation of exponential growth on glucose. The cAMP level during exponential growth on glucose is not significantly different from the level at the diauxic transition, when the glucose is depleted.

This conclusion is further supported by the results obtained with the strains displaying reduced feedback inhibition of cAMP synthesis. Such strains show a prolonged lag phase even upon resuspension of glucosegrown cells in fresh glucose medium. During this lag phase they show a huge transient hyperaccumulation of 
cAMP, confirming the conclusion that lag phase cells are subject to a strong stimulation of cAMP synthesis. Although the experimental manipulation of the cells involved a possible stress-provoking treatment (centrifugation and washing with cold water) which could lower cAPK activity, the different results obtained with the different conditions used clearly show that this treatment alone cannot be the trigger for the cAMP increase. Also with these strains the cAMP level drops again approximately concomitant with the initiation of exponential growth on glucose. A comparison of the three strains used indicates a correlation with the end of the lag phase and no correlation with the depletion of glucose from the medium. Interestingly, this transient increase in cAMP is strongly reduced in the absence of a nitrogen source and therefore seems to be truly associated with a lag phase preceding the initiation of growth, rather than the mere absence of growth.

The disappearance of glucose from the medium during the lag phase is clearly reduced in the cAPK-defective strains compared to the wild-type, but is initially very similar for the three cAPK-defective strains. Only afterwards, when substantial growth has occurred, is a correlation between growth rate and glucose depletion in the medium clearly visible. The results of the ${ }^{31} \mathrm{P}$ NMR measurements indicate normal initiation of glucose metabolism in the cAPK-defective strains. Glucose metabolism in these strains is apparently fast enough to generate the same increases in the levels of ATP and sugar phosphates as observed in wild-type cells. Important with respect to the control of the cAMP level is the fact that the cAPK-defective strains display the same increase in their intracellular $\mathrm{pH}$ as wild-type cells. This excludes the possibility that the huge transient hyperaccumulation of cAMP during the prolonged lag phase in these strains is caused by an extended period of intracellular acidification due to metabolic problems caused by the defective cAPK activity.

Our results show that simple harvesting and resuspension of exponentially growing yeast strains with defective cAPK activity in glucose-containing growth medium causes the presence of a pronounced lag phase before growth resumes. Hence, the longer lag phase of strains with attenuated cAPK activity is not specifically due to slower exit from stationary phase. This indicates that if the Ras/adenylate cyclase pathway has a function in the stimulation of outgrowth or recovery from stationary phase (Tatchell, 1993), it would also function in the re-initiation of exponential growth in cells in which exponential growth is temporarily disturbed. However, it might also be that this longer lag phase is just an artefact without true physiological relevance.

The large hyperaccumulation of cAMP during the lag phase of the cAPK-defective strains is transient. It drops back to the original level in about $1-2 \mathrm{~h}$. Since the strain with reduced, constitutive activity of cAPK lacks the $B C Y 1$-encoded regulatory subunit, the decrease in the cAMP level cannot be due to cAMP-triggered activation of cAPK with a stimulation of feedback inhibition as a result. Moreover, the $t p k 1-3 \Delta$ yak1 $1 \Delta$ strain also shows a drop in cAMP. Hence, in addition to feedback inhibition by cAPK, other mechanisms appear to exist in $S$. cerevisiae which are able to trigger potent downregulation of the cAMP level. One possibility is the socalled fermentable-growth-medium (FGM)-induced pathway for which we have suggested previously that it is able to activate the free catalytic subunits of cAPK in glucose-containing medium (Thevelein, 1991, 1994). However, other mechanisms, such as transcriptional regulation of genes involved in cAMP synthesis and/or hydrolysis cannot be excluded.

The large, transient accumulation of cAMP in the cAPKdefective strains needs a growth medium. It is clearly stimulated by the presence of glucose and a nitrogen source. On the other hand, the absence of phosphate or sulfate in the medium has little effect. The stimulation by nitrogen is only partially dependent on protein synthesis. The pronounced nitrogen effect appears at first confusing. Elaborate work with wild-type cells has clearly shown that addition of nitrogen to nitrogenstarved cells activates cAPK-controlled targets in a cAMP-independent way (Durnez et al., 1994; Hirimburegama et al., 1992; Thevelein \& Beullens, 1985). No effect of nitrogen addition on the cAMP level in nitrogenstarved cells could be observed (Hirimburegama et al., 1992). From these observations we have concluded previously that nitrogen sources do not affect cAPK targets in glucose-growing yeast cells by enhancing the cAMP level (Thevelein, 1991, 1994). In our view, the present results do not contradict this conclusion. The stimulating effect of nitrogen on the cAMP level in the cAPK-defective strains is clearly confined to the lag phase. When exponential growth on glucose starts, the cAMP level drops rapidly, whether nitrogen is present or not. This confirms that in glucose medium nitrogen does not affect cAPK targets and does not stimulate growth by stimulation of cAMP synthesis. The conclusion that nitrogen does not stimulate cAMP synthesis during growth is supported by the observation that transfer of the cells of cAPK-defective strains and wildtype strains to a nitrogen starvation medium leads to an increase in the cAMP level rather than a decrease. This result is important since wild-type cells display a phenotype indicating down-regulation of cAPK activity under such conditions. These results further support the existence of other mechanisms regulating CAPK activity besides cAMP.

In conclusion, our results indicate that in yeast cells the lag phase of growth on glucose medium is associated with a transient increase in the cAMP level. The initiation of exponential growth on glucose is associated with a decrease in CAMP, which is unrelated to the remaining glucose level in the medium. There is no significant difference in the cAMP level of cells growing exponentially on glucose and cells in the diauxic phase or growing on ethanol. These results indicate that mechanisms other than changes in the cAMP level must be responsible for the apparent changes in cAPK activity 
between cells growing in glucose and those at the diauxic shift or growing on ethanol.

\section{ACKNOWLEDGEMENTS}

We are grateful to $M$. Wigler (Cold Spring Harbor Laboratory) and J. Broach (Princeton) for the provision of yeast strains and to W. Verheyden for excellent technical assistance. This work was supported by a fellowship from the Katholieke Universiteit Leuven to P.M. and by grants from the Fund for Scientific Research - Flanders, the Belgian National Lottery, the Research Fund of the Katholieke Universiteit Leuven (GOA-action) and the European Commission (Human Capital and Mobility Programme Contract No. CHRX-CT93-0265) to J.M.T.

\section{REFERENCES}

Argüelles, J. C., Mbonyi, K., Van Aelst, L., Vanhalewyn, M., Jans, A. W. H. \& Thevelein, J. M. (1990). Absence of glucose-induced cAMP signaling in the Saccharomyces cerevisiae mutants cat 1 and cat 3 which are deficient in derepression of glucose-repressible proteins. Arch Microbiol 154, 199-205.

Belazzi, T., Wagner, A., Wieser, R., Schanz, M., Adam, G., Hartig, A. \& Ruis, H. (1991). Negative regulation of transcription of the Saccharomyces cerevisiae catalase-T (CTT1) gene by cAMP is mediated by a positive control element. EMBO J 10, 585-592.

Beullens, M., Mbonyi, K., Geerts, L., Gladines, D., Detremerie, K., Jans, A. W. H. \& Thevelein, J. M. (1988). Studies on the mechanism of the glucose-induced cAMP signal in glycolysis and glucose repression mutants of the yeast Saccharomyces cerevisiae. Eur J Biochem 172, 227-231.

Boy-Marcotte, E., Garreau, H. \& Jacquet, M. (1987). Cyclic AMP controls the switch between division cycle and resting state programs in response to ammonium availability in Saccharomyces cerevisiae. Yeast 3, 85-93.

Cameron, S., Levin, L., Zoller, M. \& Wigler, M. (1988). cAMPindependent control of sporulation, glycogen metabolism and heat shock resistance in $S$. cerevisiae. Cell 53, 555-566.

Dumont, J. E., Jauniaux, J. C. \& Roger, P. P. (1989). The cyclic AMP-mediated stimulation of cell proliferation. Trends Biochem Sci 14, 67-71.

Dumortier, F., Arguelles, J. C. \& Thevelein, J. M. (1995). Constitutive glucose-induced activation of the Ras-cAMP pathway and aberrant stationary-phase entry on a glucose-containing medium in the Saccharomyces cerevisiae glucose-repression mutant hex 2 . Microbiology 141, 1559-1566.

Durnez, P., Pernambuco, M. B., Oris, E., Argúelles, J. C., Mergelsberg, H. \& Thevelein, J. M. (1994). Activation of trehalase during growth induction by nitrogen sources in the yeast Saccharomyces cerevisiae depends on the free catalytic subunits of cAMPdependent protein kinase, but not on functional Ras proteins. Yeast 10, 1049-1064.

Engelberg, D., Perlman, R. \& Levitzki, A. (1989). Transmembrane signalling in Saccharomyces cerevisiae. Cell Signalling 1, 1-7.

Eraso, P. \& Gancedo, J. M. (1985). Use of glucose analogues to study the mechanism of glucose-mediated cAMP increase in yeast. FEBS Lett 191, 51-54.

François, J. M., Eraso, P. \& Gancedo, C. (1987). Changes in the concentration of cAMP, fructose-2,6-bisphosphate and related metabolites and enzymes in Saccharomyces cerevisiae during growth on glucose. Eur J Biochem 164, 369-373.

Gage, R. A., van Wijngaarden, W., Theuvenet, A. P. R., BorstPauwels, G. W. F. H. \& Haasnoot, C. A. G. (1984). Localization and identification of the compound causing peak ' $x$ ' in the ${ }^{31} \mathrm{P}$ NMR spectrum of Saccharomyces cerevisiae. Biochim Biophys Acta 804, 341-347.

Garret, S. \& Broach, J. (1989). Loss of Ras activity in Saccharomyces cerevisiae is suppressed by disruption of a new kinase gene, YAK1, whose product may act downstream of the cAMPdependent protein kinase. Genes Dev 3, 1336-1348.

Gibbs, J. B. \& Marshall, M. S. (1989). The ras oncogene-an important regulatory element in lower eucaryotic organisms. Microbiol Rev 53, 171-185.

Hirimburegama, K., Durnez, P., Keleman, J., Oris, E., Vergauwen, R., Mergelsberg, H. \& Thevelein, J. M. (1992). Nutrient-induced activation of trehalase in nutrient-starved cells of the yeast Saccharomyces cerevisiae: cAMP is not involved as second messenger. J Gen Microbiol 138, 2035-2043.

den Hollander, J. A., Ugurbil, K., Brown, T. R. \& Shulman, R. G. (1981). ${ }^{31} \mathrm{P}$ NMR studies of the effect of oxygen upon glycolysis in yeast. Biochemistry 20, 5871-5880.

Loureiro-Dias, M. C. \& Santos, H. (1990). Effects of ethanol on Saccharomyces cerevisiae as monitored by in vivo ${ }^{31} \mathrm{P}$ and ${ }^{13} \mathrm{C}$ nuclear magnetic resonance. Arch Microbiol 153, 384-391.

Mbonyi, K., van Aelst, L., Argüelles, J. C., Jans, A. W. H. \& Thevelein, J. M. (1990). Glucose-induced hyperaccumulation of cyclic AMP and defective glucose repression in yeast strains with reduced activity of cyclic AMP-dependent protein kinase. $\mathrm{Mol}$ Cell Biol 10, 4518-4523.

Nikawa, J., Cameron, S., Toda, T., Ferguson, K. W. \& Wigler, M. (1987). Rigorous feedback control of cAMP levels in Saccharomyces cerevisiae. Genes Dev 1, 931-937.

van der Plaat, J. B. (1974). Cyclic 3',5'-adenosine monophosphate stimulates trehalose degradation in baker's yeast. Biochem Biophys Res Commun 56, 580-587.

Russell, M., Bradshawrouse, J., Markwardt, D. \& Heideman, W. (1993). Changes in gene expression in the Ras/Adenylate cyclase system of Saccharomyces cerevisiae - correlation with cAMP levels and growth arrest. Mol Biol Cell 4, 757-765.

Santos, H. \& Turner, D. L. (1986). Characterization of the improved sensitivity obtained using a flow method for oxygenating and mixing cell suspensions in NMR. J Magn Reson 68, 345-349.

Serrano, R. (1983). In vivo glucose activation of the yeast plasma membrane ATPase. FEBS Lett 156, 11-14.

Sherman, F., Fink, G. R. \& Hicks, J. B. (1986). Methods in Yeast Genetics. Cold Spring Harbor, NY: Cold Spring Harbor Laboratory.

Tatchell, K. (1993). RAS genes in the budding yeast Saccharomyces cerevisiae. In Signal Transduction. Prokaryotic and Simple Eukaryotic Systems, pp. 147-188. Edited by J. Kurjan \& B. J. Taylor. San Diego: Academic Press.

Thevelein, J. M. (1991). Fermentable sugars and intracellular acidification as specific activators of the Ras-adenylate cyclase signalling pathway in yeast: the relationship to nutrient-induced cell cycle control. Mol Microbiol 5, 1301-1307.

Thevelein, J. M. (1994). Signal transduction in yeast. Yeast 10, 1753-1790.

Thevelein, J. M. \& Beullens, M. (1985). Cyclic AMP and the stimulation of trehalase activity in the yeast Saccharomyces cerevisiae by carbon sources, nitrogen sources and inhibitors of protein synthesis. J Gen Microbiol 131, 3199-3209.

Thevelein, J. M., Beullens, M., Honshoven, F., Hoebeeck, G., Detremerie, K., den Hollander, J. A. \& Jans, A. W. H. (1987a). Regulation of the cAMP level in the yeast Saccharomyces 
cerevisiae: intracellular $\mathrm{pH}$ and the effect of membrane depolarizing compounds. J Gen Microbiol 133, 2191-2196.

Thevelein, J. M., Beullens, M., Honshoven, F., Hoebeeck, G., Detremerie, K., Griewel, B., den Hollander, J. A. \& Jans, A. W. H. (1987b). Regulation of the cAMP level in the yeast Saccharomyces cerevisiae: the glucose-induced cAMP signal is not mediated by a transient drop in the intracellular $\mathrm{pH}$. J Gen Microbiol 133, 2197-2205.

Toda, T., Cameron, S., Sass, P., Zoller, M., Scott, J. D., McBullen, B., Hurwitz, M., Krebs, E. G. \& Wigler, M. (1987a). Cloning and characterization of $B C Y 1$, a locus encoding a regulatory subunit of the cyclic AMP-dependent protein kinase in Saccharomyces cerevisiae. Mol Cell Biol 7, 1371-1377.

Toda, T., Cameron, S., Sass, P., Zoller, M. \& Wigler, P. (1987b). Three different genes in Saccharomyces cerevisiae encode the catalytic subunits of the cAMP-dependent protein kinase. Cell 50, 277-287.

Received 4 June 1997; revised 11 July 1997; accepted 14 August 1997. 